\title{
New approaches in Quartz-Enhanced Photoacoustic Sensing
}

\author{
Angelo Sampaolo ${ }^{\mathrm{a}, \mathrm{b}}$, Pietro Patimisco ${ }^{\mathrm{a}, \mathrm{b}}$, Riccardo Pennetta ${ }^{\mathrm{a}}$, Gaetano Scamarcio ${ }^{\mathrm{a}}$, Frank K. Tittel ${ }^{\mathrm{b}}$, \\ Vincenzo Spagnolo ${ }^{\mathrm{a}, \mathrm{b}^{*}}$, \\ a Dipartimento Interateneo di Fisica, University and Politecnico of Bari, CNR-IFN UOS BARI, Via \\ Amendola 173, Bari, Italy; \\ ${ }^{b}$ Department of Electrical and Computer Engineering, Rice University, 6100 Main Street, Houston, \\ TX 77005, USA
}

\begin{abstract}
We report on the design and realization of custom quartz tuning forks with different geometries and sizes aimed to improve the photoacoustic effect in quartz-enhanced photoacoustic (QEPAS) sensor systems. A detailed analysis of the piezoelectric properties in terms of resonance frequencies, quality factors, gas damping was performed.
\end{abstract}

Keywords: Quartz tuning fork, Piezoelectric effect, Quartz enhanced photoacoustic spectroscopy

\section{INTRODUCTION}

Sensitive and selective trace gas concentrations measurements are crucial tools for the investigation and monitoring of a wide variety of physical processes in industrial and environmental monitoring, combustion processes, detection of toxic and flammable gases, as well as explosives, atmospheric science and medical breath sensing. Techniques based on laser absorption spectroscopy for trace gas sensing [1], compared to other techniques, are considerably faster with response times of $<1 \mathrm{~s}$, offer high gas specificity, capable of part-per-quadrillion (ppq) detection sensitivity [2] and permit real time in-situ measurements. One of the most robust and sensitive trace-gas optical detection techniques is photo-acoustic spectroscopy (PAS), which is capable of extremely high detection sensitivities with a compact and relatively low-cost absorption detection module (ADM) [3]. In PAS, incident optical radiation is absorbed by the gaseous medium, causes subsequent heating, which gives rise to thermal expansion of the medium. Hence, if the incident radiation is modulated in the audio frequency range the absorption results in sound generation, which can be detected using sensitive low-noise microphones. Quartz-enhanced photoacoustic spectroscopy (QEPAS) is an alternative approach to photoacoustic detection of trace gas utilizing a quartz tuning fork (QTF) as a sharply resonant acoustic transducer to detect weak photoacoustic excitation and allowing the use of extremely small volumes [4, 5]. Such an approach removes restrictions imposed on the gas cell by the acoustic resonance conditions. A quartz crystal is a natural candidate for such an application. This material is mass-produced and therefore inexpensive. Electronic watches, smartphones, digital electronics, and synchronization for asynchronous communications are built around high-Q QTFs as frequency standards. Usually QTFs with a resonant frequency of $2^{15}$ or $\sim 32,768 \mathrm{~Hz}$ are used in this application. A QTF behaves as acoustic quadrupole in which case only the anti-symmetric mode is piezoelectric active (in which case the prongs move in opposite directions). Standard QTFs possess a $\mathrm{Q} \approx 100,000$ or higher when encapsulated in vacuum and a $\mathrm{Q} \approx 10,000$ at normal atmospheric pressure. Furthermore, a QTF provides good environmental noise immunity, since the only way to cause the QTF to resonate via the photoacoustic effect is to produce sound waves from a source located between the two QTF prongs. Further enhancements of the QEPAS signal is achieved by means of a micro-resonator $(\mathrm{mR})$, if the incident sound waves are confined in the $\mathrm{mR}$. A typical acoustic $\mathrm{mR}$ consists of two thin tubes aligned either perpendicular or parallel to the QTF plane. Different QEPAS configurations have been demonstrated, such as on-beam $[4,6]$, off-beam [7], modulation cancellation [8], and evanescent wave QEPAS [9]. In Fig. 1 a schematic of the QEPAS on beam configuration for a bare QTF is shown. The laser beam is focused between the prongs of the QTF, its wavelength is modulated at the resonant frequency $\mathrm{f}_{0}$ of the QTF or $\mathrm{f}_{0} / 2$ frequency and the current signal generated via piezoelectric effect is fed via a transimpedance amplifier to a lock-in amplifier, that demodulates the QTF response at $\mathrm{f}_{0}$. Spectral data can be acquired if the laser wavelength is scanned across the selected gas absorption line.

*vincenzo.spagnolo@uniba.it; phone 39080 544-2373; fax 39080 544-2219 


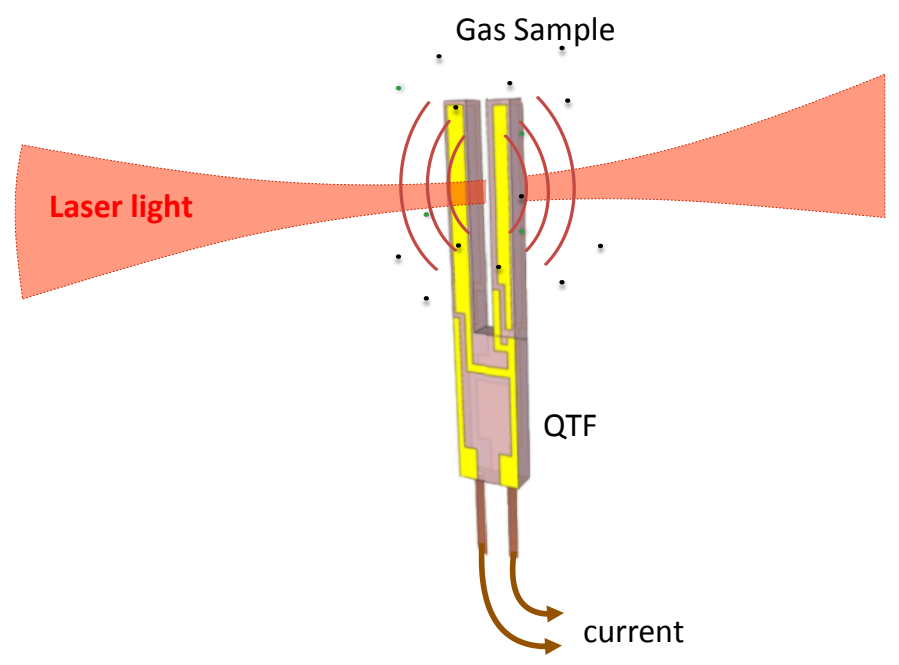

Figure 1. Sketch of an on-beam QEPAS spectrophone configuration with a bare QTF. The red circular lines mimic the acoustic wave fronts.

To date, almost all QEPAS sensors reported in literature exploit "standard" QTFs. QEPAS sensors employing standard QTFs and operating in the spectral wavelength range 1-10 $\mu \mathrm{m}$ have been demonstrated. ${ }^{5}$ However, there are two main factors that pose limitations to the use of standard QTFs:

1) QEPAS signal depends strongly on the energy relaxation rates associated with the absorption gas species. Hence, low signals are obtained when working with slowly relaxing gases such as NO or CO with a QTF having resonance frequency of $\sim 32.76 \mathrm{kHz}$. In order to increase the signal, the addition of appropriate gas relaxation promoters (such as $\mathrm{H}_{2} \mathrm{O}, \mathrm{SF}_{6}$ ) is necessary, which modify the original gas mixture sample.

2) Standard QTFs are characterized by a very small sensitive volume between their prongs $\left(\sim 0.3 \times 0.3 \times 3 \mathrm{~mm}^{3}\right)$. Furthermore, the use of light sources with a poor beam quality or emitting in the THz spectral region $(\lambda>30$ $\mu \mathrm{m})$ requires adequate focalization of the excitation beam between the prongs ( $300 \mu \mathrm{m}$ spacing). The incident radiation must not hit the QTF, as otherwise an undesirable non-zero background arises, which can be several times larger than the thermal noise level of QEPAS sensor, thereby strongly limiting the final detection sensitivity $[10,11]$.

Therefore, the use of QTFs with larger prong spacing is necessary in order to extend the QEPAS operation to poor beamquality radiation sources (LEDs) and in the THz range. Recently, the first demonstration of a QEPAS sensor employing a custom QTF having the same geometry as the standard QTF, but six times larger in size, with a space of $\sim 800 \mu \mathrm{m}$ between the prongs has been reported [12,13]. With the aim to investigated different QTF geometries in terms of spacing between the prongs, and length and thickness of the two prongs we have realized four different types of QTFs. A detailed description of the design and characterization of these innovative optoacoustic transducers follows.

\section{QTF DESIGN REALIZATION AND CHARACTERIZATION}

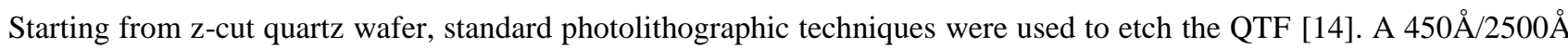
Chromium/gold layers were deposited on both sides of the QTF for electrical contacts. In table 1 are reported the main geometrical parameters. The thickness of the QTFs is $\mathrm{T}=0.25 \mathrm{~mm}$. A picture of one of QTFs realized is shown in figure 2. In order to employ these custom QTFs in a QEPAS sensing system we have to verify that they behave like a standard tuning fork transducer in terms of resonance frequency and quality factor. The basic principle of a tuning fork is well known: two prongs connected at one end make a resonator, whose resonance frequencies are defined by the properties of the composing material and by its geometry. The resonator is fixed on a holder structure. The available resonance frequencies of the tuning fork can be found analytically by considering one arm of the fork as a cantilever-vibrating prong. In the fundamental flexural mode of oscillation, the tines move in opposite directions and the center-of mass of 
the fork remains unchanged. This flexural mode vibration can be modelled by considering that each arm of the tuning fork behaves as a clamped prong.

Table 1. Dimensions of the investigated QTFs

\begin{tabular}{|c|c|c|c|c|}
\hline & QTF\#1 & QTF\#2 & QTF\#3 & QTF\#4 \\
\hline Prongs spacing $(\mathrm{mm})$ & 0,4 & 0,8 & 0,6 & 0,7 \\
\hline Prongs length L $(\mathrm{mm})$ & 3,5 & 10 & 11 & 17 \\
\hline QTF length $(\mathrm{mm})$ & 6 & 15 & 15,5 & 27 \\
\hline Prongs width W $(\mathrm{mm})$ & 0,2 & 0,9 & 0,5 & 1 \\
\hline
\end{tabular}

In this analysis, the frequency of the vibration modes is obtained by including a free-motion condition on one boundary of the prong and a clamped condition on the other (clamped-free boundary condition) and then solving for the propagation of the shear acoustic wave.

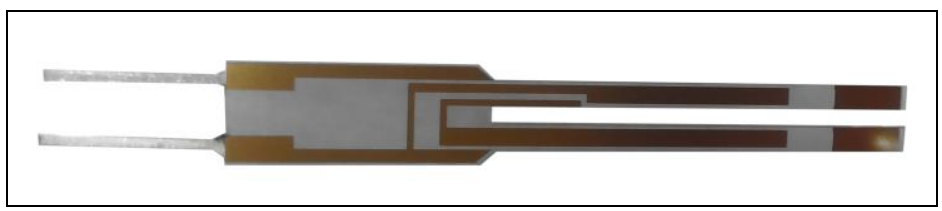

Figure 2. Picture of QTF\#4. The gold pattern allows collection of the generated piezoelectric charges. The two silver pins used for the electrical connections are also visible on the left side.

The QTF free motion is then given by the following fourth-order differential equation according to the classical EulerBernoulli theory:

$$
E I \frac{\partial^{4} y(x, t)}{\partial x^{4}}+\rho A \frac{\partial^{4} y(x, t)}{\partial t^{4}}=0
$$

where $E$ is the Young modulus of the QTF material, $I$ the moment of inertia of the prong and $A$ the cross sectional area of the prong, $\rho$ is the density of the material, $t$ is the time and $x$ and $y$ are the longitudinal and crossed directions with respect to the prong. Eq. (1) can be solved and imposing boundary conditions for the supports of the cantilever prong, the eigenfrequencies $f_{n}$ will be given by:

$$
f_{n}=\frac{\pi K}{8 L^{2}} \sqrt{\frac{E}{\rho}} n^{2}
$$

where $K=\sqrt{(I / A)}$ is the radius of gyration of the prong cross section, that for a bar with rectangular cross section, as in our case, is equal to $1 / \sqrt{12}$ times the thickness.

The other important parameter to keep in consideration is the quality factor $Q=f / \Delta f_{F W H M}$ of the resonator, where $\mathrm{f}$ is the resonant frequency and $\Delta \mathrm{f}_{\mathrm{FWHM}}$ is the full-width-half-maximum of the peak resonance profile. In order to place in evidence the dependence of the Q-factor value in vacuum from the geometrical parameter of a QTF, it is useful to consider that for small amplitude oscillations, the description of the fork as a three dimensional solid can be reduced to a single coordinate. With a sinusoidal external excitation $F \sin (\omega t)$, the equation of motion for this coordinate takes the familiar form of a driven harmonic oscillator:

$$
m \frac{d^{2} x}{d t^{2}}+b \frac{d x}{d t}+k x=F \sin (\omega t)
$$

In Eq. (3), $\omega=\sqrt{k / m}$ and $m$ is the effective mass of one prong. The effective mass depends on the fork geometry and for a rectangular cross section prong is predicted to be approximately $m=0.243 \rho_{q} V$, where $V=T \cdot W \cdot L$ is the volume, $\mathrm{T}$ the thickness, W the width and $\mathrm{L}$ the length of the prong and $\rho_{q}$ is the quartz density. The effective spring constant $k$ is proportional to the Young's modulus E [15]:

$$
k=\frac{E}{4} T\left(\frac{W}{L}\right)^{3}
$$


The effective damping constant $b$ arises from internal energy losses, which are low in pure quartz. In actual devices, additional energy loss mechanism arises, for example, from the attached electrodes and from the gas surrounding the QTF. Manufacturing variations or defects among similar QTFs have been demonstrated to strongly affect the $b$ parameter, much more than energy losses due to the quartz mass. In this case, we can correlate the $\mathrm{Q}$ factor to the geometric parameter considering that $b=m \Delta f$. Thus, ideally we have:

$$
Q=\frac{\sqrt{m k}}{b}=\frac{\sqrt{E \rho_{q}}}{4 b} \cdot\left(\frac{T W^{2}}{L}\right)
$$

This formulation allows to evaluate the effects of the geometric parameter on $\mathrm{Q}$ theoretic value in the vacuum $\left(\mathrm{Q}_{0}\right)$ and to estimate the damping parameter $b$.

From Eq. (2) as well as substituting the geometrical and the elastic (with a Young modulus of quartz, $\mathrm{E}=0.72 \cdot 10^{11} \mathrm{~N} / \mathrm{m}^{2}$, and $\rho_{\mathrm{q}}=2650 \mathrm{Kg} / \mathrm{m}^{3}$ its density) parameters, we obtain solutions for the first two flexural modes $(\mathrm{n}=1,3)$ shown in Table 1.

Table 2. Calculated QTF eigenfrequencies corresponding to the first two flexural order modes.

\begin{tabular}{|c|c|c|}
\hline QTF & $f_{l}$ & $f_{3}$ \\
\hline$\# 1$ & 13747 & 86089 \\
\hline$\# 2$ & 7578 & 47457 \\
\hline$\# 3$ & 3479 & 21789 \\
\hline$\# 4$ & 2913 & 18245 \\
\hline
\end{tabular}

In Fig. 3 are shown two snapshots, which mimic the correlated QTF motion at $f_{l}$ and $f_{3}$.

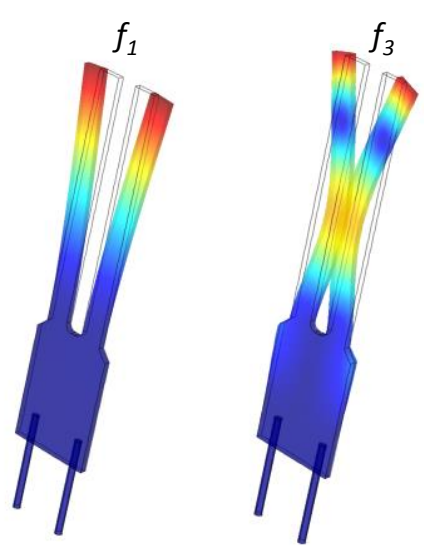

Figure 3. FEMLAB simulated snapshots of the QTF motion for the first two flexural modes $(n=1,3)$.

The characterization of the QTFs has been carried out by feeding them with a sinusoidal voltage excitation. A wavefunction generator connected to a trans-impedance preamplifier stage (x30) allowed us to perform a frequency scan near the QTF resonance. The electric response of the resonators have been recorded by means of a lock-in amplifier referred to the voltage excitation frequency in a wide range of air pressure (from a few Torr to atmospheric pressure) using a pressure-controlled chamber in which QTFs were inserted. In Fig. 4 shows the data related to the first resonant modes measured at 50 Torr for the four investigated QTFs. 

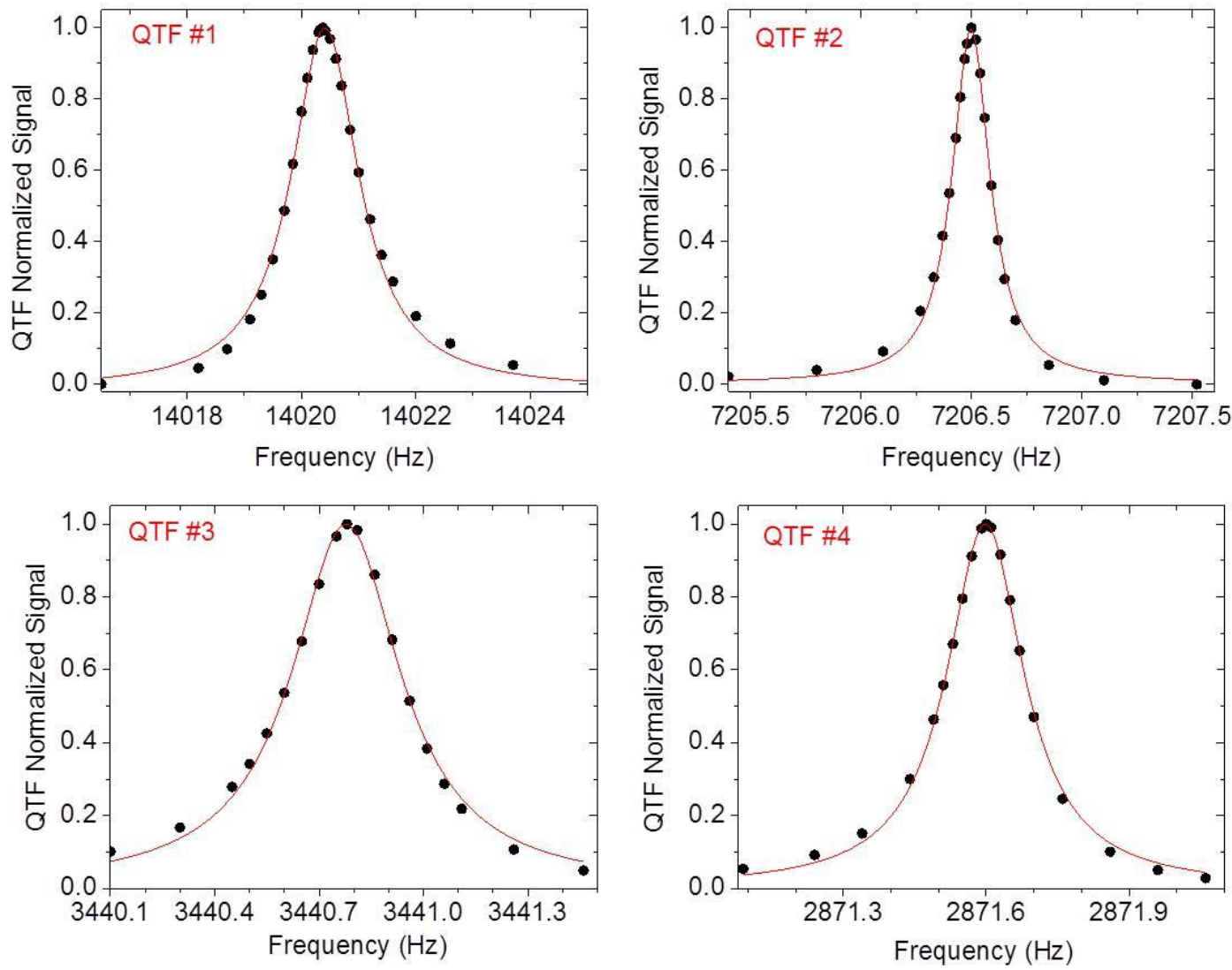

Figure 4. Frequency profiles for $f_{1}$ of four QTFs measured at 50 Torr in air. The solid red lines are the Lorentzian best fits.

By fitting the resonance curves with Lorentzian functions, we can extract the resonance frequencies and the corresponding quality factor $Q$. The value obtained at 50 Torr are reported in Table 3 . The small discrepancies from the $f_{l}$ theoretical values, calculated under vacuum conditions (see Table 1), are due to damping effects by the ambient gas, dependence of the elasticity modulus of quartz on the crystallographic axes orientation and deviations in geometry between the modeled and the real QTF.

Table 3. Resonant frequencies and Q-factor measured at $\mathrm{P}=50$ Torr in ambient air

\begin{tabular}{|c|c|c|}
\hline QTF & Measured $f_{1}(\mathrm{~Hz})$ & Q-factor @ 50 \\
\hline$\# 1$ & 14021 & 8500 \\
\hline$\# 2$ & 7207 & 33300 \\
\hline$\# 3$ & 3441 & 9300 \\
\hline$\# 4$ & 2872 & 14620 \\
\hline
\end{tabular}

Effectively, the presence of damping due to the viscosity of a fluid medium surrounding the QTF modifies eq. (1) as follows:

$$
E I \frac{\partial^{4} y(x, t)}{\partial x^{4}}+C_{d} \frac{\partial y(x, t)}{\partial t}+(\rho A+u) \frac{\partial^{4} y(x, t)}{\partial t^{4}}=0
$$

in which $C_{d}$ is the damping parameter due to the presence of a gas and $u$ is the added mass per unit length, proportional to $\rho_{q}$ and thus the pressure of the fluid medium. Consequently, the resonance frequency decreases with increasing gas pressure as: 


$$
\Delta f=\frac{f_{n}-f^{\prime} n}{f^{\prime} n}=-\frac{1}{2} \frac{u}{\rho A}
$$

The damping parameter depends on the pressure as $C_{d}=\mathrm{a} \sqrt{\mathrm{P}}$, where a is a parameter specific for the tuning fork. The influence of the fluid damping on the Q-values can be expressed in terms of the energy loss. Thus, $\mathrm{Q}(\mathrm{P})$ can be described as [13]:

$$
Q=\frac{Q_{0}}{1+Q_{0} \cdot a \cdot \sqrt{P}}
$$

where $Q_{0}$ is the quality factor under vacuum condition. In order to determine this parameter we measured the Q-factor as a function of the pressure behavior of the four QTFs. The results are shown in table 4 where we report also the calculated $b$ parameters by using Eq. 5 and the estimated $Q_{0}$-values. Note that these $b$ values are related to the damping of the QTFs under vacuum conditions, and thus only to their mechanical properties (and not to gas damping effect). As expected the bigger the size of the QTF, the higher the damping parameter in vacuum.

Table 4. Estimated Q-factor under vacuum and damping constant $b$.

\begin{tabular}{|c|c|c|}
\hline QTF & Q & $b(\mathrm{Kg} / \mathrm{s})$ \\
\hline$\# 1$ & 18990 & 14.35 \\
\hline$\# 2$ & 70050 & 27.58 \\
\hline$\# 3$ & 17110 & 31.68 \\
\hline$\# 4$ & 29614 & 47.37 \\
\hline
\end{tabular}

The results obtained from the custom QTF characterization demonstrate that they behave like tuning fork resonators with $\mathrm{Q}_{0}$ values of tens of thousands, thus they can be employed for QEPAS-based gas sensing applications.

\section{CONCLUSIONS}

The realization of four custom tuning fork designs with different geometries and sizes was demonstrated for use in QEPAS-based trace gas sensors with improved sensing performance. We increased the QTF prong spacing with respect to a standard QTF up to $800 \mu \mathrm{m}$ with the aim to use QTF also with poor beam-quality sources (LEDs) and THz laser sources. In addition, we have reduced the QTFs resonance frequency in the range $2.8-14 \mathrm{KHz}$ so that they can be employed with slow relaxing gases without addition of a relaxation promoting gas under stable flow conditions. The next step will be the testing and performance evaluation of the reported new QTFs with reference gases such as ethylene, and nitric oxide. The subsequent design and realization of a micro-resonator system should result in an enhancement of the QEPAS detection sensitivity by at least a factor of magnitude [16].

\section{ACKNOWLEDGEMENTS}

The authors gratefully acknowledge financial support from three Italian research projects: PON01 02238, PON02 00675 and PON02 00576.

\section{REFERENCES}

[1] Hodgkinson, J., Tatam, R.P., ”Optical gas sensing: A review, “Meas. Sci. Technol. 24, 012004 (2013).

[2] Galli, I., Bartalini, S., Borri, S., Cancio, P., Mazzotti, D., De Natale, P., Giusfredi, G., ’Molecular Gas Sensing Below Parts Per Trillion: Radiocarbon-Dioxide Optical Detection," Phys. Rev. Lett. 107, 270802 (2011).

[3] Elia, A., Lugarà, P.M., Di Franco, C., Spagnolo, V., "Photoacoustic Techniques for Trace Gas Sensing Based on Semiconductor Laser Sources, “ Sensors 9, 9616-9628 (2009).

[4] Kosterev, A.A., Bakhirkin, Y.A., Curl, R.F., Tittel, F.K., “Quartz-enhanced photoacoustic spectroscopy,” Opt. Lett. 27, 1902-1904 (2002)

[5] Patimisco, P., Scamarcio, G., Tittel, F.K., Spagnolo, V., "Quartz-Enhanced Photoacoustic Spectroscopy: A Review," Sensors 14, 6165 (2014). 
[6] Kosterev, A.A, Tittel, F.K., Serebryakov, D., Malinovsky, A.L., Morozov, I., “Applications of quartz tuning forks in spectroscopic gas sensing," Rev. Sci. Instr., 76, 043105 (2005).

[7] Liu, K., Guo, X., Yi, H., Chen, W., Zhang, W., Gao, X., "Off-beam quartz-enhanced photoacoustic spectroscopy," Opt. Lett. 34, 1594 (2009).

[8] Spagnolo, V., Dong, L., Kosterev, A.A., Tittel, F.K., "Modulation cancellation method for isotope ${ }^{18} \mathrm{O} /{ }^{16} \mathrm{O}$ ratio measurements in water," Opt. Expr. 20, 3401-3407 (2012).

[9] Cao, Y., Jin, W., Ho, L.H., Liu, Z., "Evanescent-wave photoacoustic spectroscopy with optical micro/nano fibers," Opt. Lett. 37, 214-216 (2012).

[10] Spagnolo, V., Kosterev, A.A., Dong, L., Lewicki, R., Tittel, F.K., "NO trace gas sensor based on quartzenhanced photoacoustic spectroscopy and external cavity quantum cascade laser,“ Appl. Phys. B, 100, 125-130 (2010)

[11]Dong, L., Spagnolo, V., Kosterev, A.A., Lewicki, R., Tittel, F.K, "Ppb-level detection of nitric oxide using an external cavity quantum cascade laser based QEPAS sensor," Opt. Expr. 19, 24037-24045 (2011).

[12] Borri, S., Patimisco, P., Sampaolo, A., Beere, H.E., Ritchie, D.A., Vitiello, M.S., Scamarcio, G., Spagnolo, V., "Terahertz quartz enhanced photo-acoustic sensor," Appl. Phys. Lett. 103, 021105:1-021105:4 (2013).

[13] Patimisco, P., Borri, S., Sampaolo, A., Beere, H.E., Ritchie, D.A., Vitiello, M.S., Scamarcio, G., Spagnolo, V., "A quartz enhanced photo-acoustic gas sensor based on a custom tuning fork and a terahertz quantum cascade laser," Analyst 139, 2079 (2014).

[14]Lee, S., Lee, J.Y., Park, T.S., "Fabrication of SMD 32.768 kHz tuning fork-type crystals: Photolithography and selective etching of an array of quartz tuning fork resonators," Materials and Corrosion 52, $712-715$ (2001).

[15] Oria, R., Otero, J., Gonzales, L., Botaya, L., Carmona, M., Puig-Vidal, M., "Finite element analysis of electrically excited quartz tuning fork devices" Sensors 13, 7156-7169 (2013).

[16] Dong, L., Kosterev, A.A., Thomazy, D., Tittel, F.K., “QEPAS spectrophones: Design, optimization, and performance," Appl. Phys. B 100, 627-635 (2010). 